\title{
VATS Versus Open Surgery for Lung Cancer Resection: Moving Beyond the Incision
}

\author{
Aaron M. Cheng, MD, and Douglas E. Wood, MD
}

\begin{abstract}
Surgery remains the primary therapy in the treatment of earlystage lung cancer. Traditionally, anatomic resection via open thoracotomy has been the conventional approach, but as experience with minimally invasive lung surgery has increased, video-assisted thoracoscopic surgical (VATS) lobectomy is being performed more commonly for treatment of lung cancer. Proponents of VATS have argued that thoracoscopic resection for lung cancer is not only safe but is also superior to the open approach. VATS enthusiasts even have proposed that this approach should be the standard of care and a metric for quality in lung cancer surgery. Such zeal for promoting a "preferred" technique, however, obscures focus from other time-proven, but perhaps less fashionable, factors that have a tremendous impact on quality and lung cancer outcomes, namely cancer staging and quality of cancer surgery. Rather than debate incisions, thoracic surgeons should advocate for specialty care and surgical quality that assures the best short- and long-term outcomes for patients, regardless of the surgical approach. (J Natl Compr Canc Netw 2015;13:166-170)
\end{abstract}

Surgery has remained the primary therapy in the treatment of early-stage lung cancer. Traditionally, this has meant anatomic resection by open thoracotomy. Since the initial report of video-assisted thoracoscopic surgical (VATS) lobectomy performed by McKenna, ${ }^{1}$ early proponents of VATS sought to demonstrate that this approach for lung cancer surgery was feasible, safe, and comparable to the open technique. As the experience with minimally invasive surgery has grown and the acceptance of VATS has expanded, proponents have ar-

From the Division of Cardiothoracic Surgery, University of Washington, Seattle, Washington.

Submitted April 9, 2014; accepted for publication October 2, 2014.

The authors have disclosed that they have no financial interests,

arrangements, affiliations, or commercial interests with the

manufacturers of any products discussed in this article or their

competitors.

Correspondence: Douglas E. Wood, MD, Division of Cardiothoracic

Surgery, University of Washington, 1959 NE Pacific Street, Box

356310, Seattle, WA 98159-6310. E-mail: dewood@u.washington.edu gued that thoracoscopic resection for lung cancer is not only safe but is superior to thoracotomy, and further argue that VATS should be standard of care and a metric of quality for lung cancer surgery. However well intended these enthusiasts are in promoting VATS as the "preferred" technique for lung cancer resection, their focus on a relatively trivial technical comparison obscures other time-proven, but perhaps less fashionable, factors that have a huge impact on quality and cancer outcomes, namely cancer staging and quality of cancer surgery. Rather than debating incisions, thoracic surgeons should be advocating for specialty care and surgical quality that assures the best short- and long-term outcomes for patients, regardless of surgical approach.

Innumerable published reports now describe improved short-term functional recovery, less incisional pain, shorter chest tube duration, and shorter hospital length of stay in patients undergoing VATS compared with traditional thoracotomy. ${ }^{2,3}$ These studies, however, have frequently compared outcomes of VATS resection with those of historical open controls, which traditionally has been a posterolateral approach and fails to account for the concomitant advances in adjunctive therapies and alternative muscle-sparing techniques often used with contemporary open approaches to anatomic pulmonary resections. ${ }^{4,5}$ Most notably, perioperative pain management using different techniques of regional analgesia has been shown to significantly reduce postoperative morbidity and complications after pulmonary resection, and it is conceivable that the implementation—or lack —of a strategic approach to address acute postoperative pain after thoracic surgery impacts a patient's recovery, pain perception, and hospital length of stay more so than the specific surgical approach. ${ }^{6-8}$ Similarly, studies directly comparing the VATS approach with a limited thoracotomy approach, such as an anteroaxillary or anterolateral muscle-sparing technique, 
which also has been shown to improve functional recovery compared with the traditional posterolateral approach, are also lacking. ${ }^{9,10}$

The debate between the merits of VATS promotes lively, if not proselytizing, discussion at thoracic surgical meetings. And the nearly religious fervor with which VATS enthusiasts advocate technique over other important metrics of quality leaves little room for others to express equipoise regarding oncologic outcomes or to recognize associated factors that may explain many of the perceived benefits of VATS.

Undoubtedly, far more important factors in improving disease-free survival for patients with lung cancer are appropriate and meticulous staging, proper treatment selection, multidisciplinary delivery of care, and surgeon expertise rather than the actual incisional approach. VATS has provided a large number of tremendous technical and patient management advances that have allowed for improvements in care for many patients, including those undergoing resection for lung cancer. However, the conclusion that VATS should be the sole or even primary technique for most lung cancer resections is myopic and does not consider true limitations in the data regarding VATS, simultaneous (and even VATSrelated) improvements in open surgical technique, and factors relating to extent of staging and volume/ specialty-related outcomes that are likely much more powerful predictors of quality in lung cancer surgery.

Several retrospective studies and single-center experiences have been published that describe decreased perioperative morbidity associated with VATS over thoracotomy. However, in the absence of randomized data, each of these trials suffers from both selection bias and publication bias, undermining the credibility of the reported outcomes. One of the largest recent studies comparing these 2 approaches was a meta-analysis of several studies, comprising a total of more than 6000 patients published by Whitson et $\mathrm{a}^{11}$ in 2008 . The authors analyzed data from 39 articles to derive an overall complication rate of $16 \%$ for VATS compared with $31 \%$ for open lobectomy. In addition, chest tube duration and total hospital length of stay were notably shorter with the VATS approach compared with the open technique: 4.2 versus 5.7 days and 8.3 versus 13.3 days, respectively. However, the authors' summation of multiple retrospective single-center case series only magnifies the publication and patient selection biases that have consistently been criticisms of these nonrandomized reports. In contrast, others have shown no differences in qualityof-life outcomes when evaluating patient-reported pain scales, and a trend toward worse mental component scores in the VATS arm after 1 year. ${ }^{12}$ The conspicuous absence of well-designed, prospective, randomized trials directly comparing minimally invasive lung cancer resection with the open approach highlights the intense sentiment that VATS advocates maintain, arguing that a randomized trial is "unethical" because of the "clear advantages of VATS resection." Yet, this contention undermines the credibility of VATS results, whereas a randomized trial allows for the true correction of both known and unknown variables that influence outcome and for the clarification of important factors of patient selection.

Without prospective patient randomization, the decisions that bias one patient to undergo a VATS and another to undergo the open approach are not taken into account. One obvious example is the fact that VATS lobectomies are performed more often in patients with smaller and more peripheral tumors, and that this variability even occurs within a given stage of cancer and is therefore not "stage-corrected" in reported studies. Several other obvious selection biases exist, and undoubtedly, many are not readily identified or corrected for by surgeons reporting their VATS outcomes. A second type of publication bias is that surgeons reporting their VATS experience are nearly exclusively highly specialized, board-certified, high-volume, academic surgeons who likewise would show better surgical outcomes in any comparison with national or historical results in lung cancer surgery. ${ }^{13}$ Further, it is well-known that evaluation of new technology or techniques favors publication of those with good outcomes or "positive" results, and that those with less favorable results remain silent, resulting in a possible deceptive skewing of perceived clinical outcomes.

Despite the increasing prevalence of VATS resection for lung cancer surgery among the general thoracic surgical community, a significantly higher proportion of VATS procedures continue to be performed by experienced thoracic surgeons practicing at high-volume and academic medical centers than the community at large. An example of the publication bias is clear from an important noninferiority analysis by Farjah et $\mathrm{al}^{14}$; although an overall comparison of VATS with thoracotomy showed no worse morbidity or mortality associated with VATS, a higher hazard for death was, in fact, noted with the 
Cheng and Wood

VATS approach compared with open surgery when performed by lower-volume surgeons at lower-volume, nonteaching institutions. This implies that the benefit of VATS may be significantly, or even predominantly, due to the surgeon, rather than the surgical incision, as has been noted in other critiques of the pro-VATS literature. ${ }^{15}$ Even more importantly, this identifies an area of major limitation in the argument from VATS proponents who feel that most anatomically appropriate lung cancers should be resected using VATS techniques. In fact, promotion of this policy may result in substantially worse outcomes, because many surgeons may not be as adept at VATS procedures, may have individually better outcomes with open surgery, or even just have a technical preference for thoracotomy for a wide variety of technical and/or oncologic reasons. Despite the enthusiasm by VATS enthusiasts, and a push to define VATS lobectomy as the standard of care for early-stage tumors, the reality is that only $58 \%$ of recent cardiothoracic surgical graduates felt proficient to perform VATS lobectomies. ${ }^{16}$ In fact, even at academic medical centers where presumably lung cancer surgery is predominantly subspecialty focused, general thoracic surgeons reported only averaging 16 VATS lobectomies per year. ${ }^{17}$

The development of VATS techniques has had collateral benefits, particularly in appreciating the role of different approaches to pulmonary dissection and in challenging traditionally dogma regarding postoperative patient management. Conventional open lobectomy is performed by pulmonary dissection occurring along the lung fissures, whereas VATS surgeons often use a "fissure-less lobectomy" approach. ${ }^{18}$ Fissure dissection in patients with poorly developed lung fissures can increase chest tube duration due to air leaks, which adds to increased length of hospitalization; however, adopting a "fissure-less approach" in the open technique has also been demonstrated to reduce chest tube duration and length of stay, ${ }^{19,20}$ a technique borrowed from VATS that also may improve outcomes in open surgery. Of even greater significance, the advances in standardizing perioperative care pathways associated with the development of VATS procedures have benefited patients with lung cancer who undergo either approach for surgery. These changes have challenged traditional assumptions regarding chest tube requirements and management that have led to earlier tube removal, promoted preemptive pain management and early mobility protocols, and recognized the value of setting clear expectations regarding discharge timing and postoperative instructions for patients and their families. The concomitant implementation of fast-track protocols after anatomic lung resection and after the VATS approach confounds any direct attribution of the benefits in postoperative recovery to the VATS approach alone. ${ }^{21,22}$ For instance, the thoracic surgical group from Brazil reported that they were able to discharge $89.9 \%$ of their patients after open lobectomy for lung cancer on postoperative day 2 by following a comprehensive fast-track rehabilitation protocol..$^{23}$ Other authors have also reported similar shortened hospital lengths of stay for patients after open lung lobectomies when using clinical pathways for major lung resection. ${ }^{24}$ Whether the shorter hospitalizations observed with VATS procedures represent contributions of the minimally invasive approach or are the result of parallel advances in associated perioperative care that have been championed by VATS advocates cannot be answered. Likely they are the result of both, but it would be misleading not to acknowledge the "halo effect" of these fast-track pathways in reducing hospital stay, independent of the procedure type.

Although some proponents of the VATS approach have even alleged improved cancer survival, no evidence indicates that one treatment approach is oncologically superior to the other. The only prospective randomized trial comparing the oncologic results of VATS with open lobectomy has been a small Japanese study that enrolled patients to receive VATS or open resection for clinical stage IA lung cancer. ${ }^{25}$ This study found no difference in survival between VATS resection or open thoracotomy. More recently, some studies have used propensity score-matched analysis and large database samples to compare the oncologic outcomes of lobectomy using VATS versus thoracotomy and have not demonstrated superiority of cancer outcomes related to thoracoscopic lobectomy. ${ }^{26,27}$ A recent propensity score matched analysis of VATS compared with open lobectomy was reported of patients enrolled in the American College of Surgeons Oncology Group Z0030/Alliance trial. A subset analysis found no differences between the groups in terms of overall survival, disease-free survival, or the development of a new primary tumor. ${ }^{28}$

What is known to have a profound impact on lung cancer survival is accurate staging. Because staging 
drives treatment decisions, inaccurate staging can deny treatment modalities for patients who otherwise would gain a survival benefit from intervention. Although the NCCN Clinical Practice Guidelines in Oncology (NCCN Guidelines) for Non-Small Cell Lung Cancer recommend invasive mediastinal staging for most patients, including those with early-stage cancer, ${ }^{29}$ remarkably only $28 \%$ of lung cancer resections are preceded by mediastinoscopy (or more recently endobronchial ultrasound biopsy), and even more staggering is that, of the mediastinoscopies performed in the United States, only $50 \%$ include pathologic nodal specimens. ${ }^{30}$ This poor adherence to current guidelines is caused by a lack of specialized surgical care and a frequent disregard of guidelines, even by board-certified thoracic surgeons. More than surgical incision, significant improvements in lung cancer outcomes could be accomplished by referral of patients to "board-certified thoracic surgeons who perform lung cancer surgery as a prominent part of their practice," as noted in the current NCCN Guidelines (to view the most recent version of these guidelines, visit NCCN.org). ${ }^{29}$ Process-of-care initiatives, such as the American College of Surgeons Commission on Cancer ProvenCare Lung Cancer Collaborative, provide other opportunities for improved patient outcomes. ${ }^{31}$

Although preresection mediastinal staging is paramount for identifying patients who are optimally treated with neoadjuvant therapy before surgery, or who should not be treated with surgery at all, intraoperative lymph node staging is also critical for determining which patients should be considered for adjuvant chemotherapy. Several recent studies have demonstrated a concern regarding adequacy of lymph node resection and diminished pathologic upstaging with VATS resection. Using the The Society of Thoracic Surgeons General Thoracic Surgery database, Boffa et $\mathrm{al}^{32}$ reported a statistically significant lower N0 to N1 upstaging in their study of nearly 4400 patients with clinical stage I lung cancer who had undergone VATS anatomic resection compared with the 7137 patients who underwent open surgery. ${ }^{32}$ Similar findings have also been published by several other highly regarded thoracic surgical oncologists. ${ }^{33-35}$ In each of these studies, the authors separately found that fewer lymph nodes were being sampled and evaluated using the VATS approach compared with open lobectomy. Although these studies did not find statistical differences in rates of survival between the 2 surgical approaches, patients who are understaged may not receive the appropriate adjuvant therapy, and this is likely to result in survival differences now that adjuvant therapy has become standard of care for resected stage II or greater lung cancer. The NCCN Guidelines for NonSmall Cell Lung Cancer indicate that at least 3 mediastinal lymph node stations should be assessed in patients undergoing lobectomy, irrespective of approach. ${ }^{29}$ From a practical standpoint, those who champion a VATSonly approach must ensure that VATS lymph node staging is being performed as well as in the open approach.

Finally, although academic thoracic surgeons debate the benefits of a VATS versus a thoracotomy approach to pulmonary resection, it seems that this is missing the forest for the trees and actually focusing on the wrong debate. In the United States, $25 \%$ to $50 \%$ of lung cancer resections are performed by nonspecialist surgeons (ie, those without formal training or board certification in cardiothoracic surgery). ${ }^{36-38}$ This statistic is staggering compared with the rest of the developed world where specialist surgeons exclusively care for these patients. And extensive data now exist outlining improved outcomes for patients undergoing lung cancer surgery by specialist surgeons, showing lower morbidity and mortality, improved long-term cancer and overall survival, and decreased cost. ${ }^{36-38}$ Therefore, one might seek to reframe the argument to one focusing on who is doing the surgery rather than what incision is being used. Experienced and well-trained surgeons are in the best position, both to have experience with VATS approaches and to best consider which patients and operations will be better served by a VATS or an open surgery. And these same surgeons are best equipped to provide complete staging and appropriate multidisciplinary decisions in oncology care, and to achieve the best short- and long-term outcomes for patients with lung cancer. The important issue is not the approach, but who does it, and assuring highquality care for all patients, whether they undergo VATS surgery or an open thoracotomy.

How does all of this fit into the context of the debate over which surgical approach is superiorVATS versus open-for the treatment of early stage non-small cell lung cancer? The overwhelming number of published studies suggesting the noninferiority of VATS to open lobectomy are from experienced thoracic surgical oncologists performing VATS lobectomies at high-volume centers, which undoubtedly also have well-established multidisciplinary thoracic oncology teams. The excellent out- 
Cheng and Wood

comes that have been achieved with VATS lobectomy are arguably more representative of the skill, judgment, and expertise of these high-performance surgeons than of any intrinsic oncologic superiority of the VATS approach. Thoracic surgeons should focus their efforts on what really matters-promoting the oncologic effectiveness of surgical resection in the management of lung cancer-rather than on the incisional approach. The extent of anatomic resection and the thorough assessment of lymph nodes, whether performed using VATS or thoracotomy, remain central to oncologic cure and effective treatment. Rather than view these surgical approaches as competing and exclusive, the rational perspective is to appreciate that the right oncologic choice depends much more on patient characteristics, gross tumor profile, and individual surgeon expertise and experience than on the length of the incision.

\section{References}

1. McKenna RJ Jr. Lobectomy by video-assisted thoracic surgery with mediastinal node sampling for lung cancer. J Thorac Cardiovas Surg 1994;107:879-882.

2. Flores RM, Park BJ, Dycoco J, et al. Lobectomy by video-assisted thoracic surgery (VATS) versus thoracotomy for lung cancer. J Thorac Cardiovasc Surg 2009;138:11-18.

3. Villamizar NR, Darrabie MD, Burfeind WR, et al. Thoracoscopic lobectomy is associated with lower morbidity compared with thoracotomy. J Thorac Cardiovasc Surg 2009;138:419-425.

4. Kim AW, Detterbeck FC. Innovations in thoracic surgery. Curr Opin Anesthesiol 2013;26:13-19.

5. Jones NL, Edmonds L, Ghosh S, Klein AA. A review of enhanced recovery for thoracic anaesthesia and surgery. Anesthesia 2013;68:179-189.

6. Maxwell C, Nicoara A. New developments in the treatment of acute pain after thoracic surgery. Curr Opin Anesthesiol 2014;27:6-11.

7. Baidya Dk, Khanna P, Maitra S. Analgesic efficacy and safety of thoracic paravertebral and epidural analgesia for thoracic surgery: a systematic review and meta-analysis. Interact Cardiovasc Thorac Surg 2014;18:626-636.

8. Elsayed H, McKevith J, McShane J, et al. Thoracic epidural or paravertebral catheter for analgesia after lung resection: is the outcome different? J Cardiothorac Vasc Anesth 2012;26:78-82.

9. Nosotti M, Baisi A, Mendogni P, et al. Muscle sparing versus posterolateral thoracotomy for pulmonary lobectomy: randomized controlled trial. Interact Cardiovasc Thorac Surg 2010;11:415-419.

10. Nomori $\mathrm{H}$, Horio $\mathrm{H}$, Naruke $\mathrm{T}$, et al. What is the advantage of thorascopic lobectomy over a limited thoracotomy procedure for lung cancer surgery? Ann Thorac Surg 2001;72:879-884.

11. Whitson BA, Groth SS, Duval SJ, et al. Surgery for early-stage non-small cell lung cancer: a systematic review of the video-assisted thoracoscopic surgery versus thoracotomy approaches to lobectomy. Ann Thorac Surg 2008;86:2008-2018.

12. Rizk NP, Ghanie A, Hsu M, et al. A prospective trial comparing pain and quality of life measures after anatomic lung resection using either thoracoscopy or thoracotomy. Ann Thorac Surg 2014;98:1160-1166.

13. Park HS, Detterbeck FC, Boffa DJ, et al. Impact of hospital volume of thoracoscopic lobectomy on primary lung cancer outcomes. Ann Thorac Surg 2012;93:372-380.

14. Farjah F, Wood DE, Mulligan MS, et al. Safety and efficacy of video-assisted versus conventional lung resection for lung cancer. J Thorac Cardiovasc Surg 2009;137:1415-1421

15. Wood DE. What is most important in improving outcomes after pulmonary lobectomy: the surgeon or the approach? Eur J Cardiothorac Surg 2013;43:817-819.

16. Boffa DJ, Gangadharan S, Kent M, et al. Self-perceived video-assisted thoracic surgery lobectomy proficiency by recent graduates of North
American thoracic residencies. Interact Cardiovasc Thorac Surg 2012;14:797-800.

17. Cooke DT, Wisner DH. Who performs complex noncardiac thoracic surgery in United States academic medical centers? Ann Thorac Surg 2012;94:1060-1064

18. Balsara KR, Balderson SS, D'Amico TA. Surgical techniques to avoid parenchymal lung injury during lung resection (fissureless lobectomy) Thorac Surg Clin 2010;20:365-369.

19. Ng T, Ryder BA, Machan JT, et al. Decreasing the incidence of prolonged air leak after right upper lobectomy with the anterior fissureless technique. J Thorac Cardiovasc Surg 2010;139:1007-1011.

20. Gómez-Caro A, Calvo MJ, Lanzas JT, et al. The approach of fused fissures with fissureless technique decreases the incidence of persistent air leak after lobectomy. Eur J Cardiothorac Surg 2007;31:203-208.

21. Muehling BM, Halter GL, Schelzig H, et al. Reduction of postoperative pulmonary complications after lung surgery using a fast track clinical pathway. Eur J Cardiothorac Surg 2008;34:174-180.

22. McKenna RJ Jr, Mahtabifard A, Pickens A, et al. Fast-tracking after video-assisted thoracoscopic surgery lobectomy, segementectomy, and pneumonectomy. Ann Thorac Surg 2007;84:1663-1668.

23. Das-Neves-Pereira JC, Bagan P, Coimbra-Israel AP, et al. Fast track rehabilitation for lung cancer lobectomy: a five-year experience. Eur ] Cardiothorac Surg 2009;36:383-392.

24. Tovar EA, Roethe RA, Weissig MD, et al. One-day admission for lung lobectomy: an incidental result of a clinical pathway. Ann Thorac Surg 1998;65:803-806

25. Sugi K, Kaneda Y, Esato K. Video-assisted thoracoscopic lobectomy achieves a satisfactory long-term prognosis in patients with clinical stage IA lung cancer. World J Surg 2000;24:27-31.

26. Paul S, Sedrakyan A, Chiu Y, et al. Outcomes after lobectomy using thoracoscopy vs thoracotomy. Eur J Cardiothorac Surg 2013;43:813-817.

27. Stephens N, Rice D, Correa A, et al. Thoracoscopic lobectomy is associated with improved short-term and equivalent oncological outcomes compared with open lobectomy for clinical stage I non-small-cell lung cancer: a propensity-matched analysis of 963 cases. Eur J Cardiothorac Surg 2014:46:607-613

28. Su S, Scott WJ, Allen MS, et al. Patterns of survival and recurrence after surgical treatment of early stage non-small cell lung carcinoma in the ACOSOG Z00300 (ALLIANCE) trial. J Thorac Cardiovasc Surg 2014;147:747-753.

29. Ettinger DS, Wood DE, Akerley W, et al. NCCN Clinical Practice Guidelines in Oncology: Non-Small Cell Lung Cancer. Version 2, 2013 Available at: NCCN.org. Accessed January 13, 2015.

30. Little AG, Rusch VR, Bonner JA, et al. Patterns of surgical care of lung cancer patients. Ann Thorac Surg 2005;80:2051-2056.

31. Katlic MR, Facktor MA, Berry SA, et al. ProvenCare Lung Cancer: a multiinstitutional collaborative. CA Cancer J Clin 2011;61:382-396.

32. Boffa DJ, Kosinski AS, Subroto P, et al. Lymph node evaluation by open or video-assisted approaches in 11,500 anatomic lung cancer resections. Ann Thorac Surg 2012;94:347-353.

33. Lee PC, Nasar A, Port JL, et al. Long-term survival after lobectomy for nonsmall cell lung cancer by video-assisted thoracic surgery versus thoracotomy Ann Thorac Surg 2013;96:951-961.

34. Denlinger CE, Fernandez F, Meyers BF, et al. Lymph node evaluation by video-assisted thoracoscopic lobectomy versus lobectomy by thoracotomy. Ann Thorac Surg 2010;89:1730-1736.

35. Merritt RE, Hoang CD, Shrager JB. Lymph node evaluation achieved by open lobectomy compared with thoracoscopic lobectomy for NO lung cancer. Ann Thorac Surg 2013;96:1171-1177.

36. Farjah F, Flum DR, Varghese TK, et al. Surgeon specialty and long-term survival after pulmonary resection for lung cancer. Ann Thorac Surg 2009;87:995-1006.

37. Schipper PH, Diggs BS, Underleider RM, Welke KF. The influence of surgeon specialty on outcomes in general thoracic surgery: a national sample 1996 to 2005. Ann Thorac Surg 2009;88:1566-1573.

38. Wood DE, Farjah F. Surgeon specialty is associated with better outcomes: the facts speak for themselves. Ann Thorac Surg 2009;88:1393-1395. 